\title{
Análise de evidências sobre o conhecimento dos riscos de queda em pacientes
}

\section{hospitalizados}

\author{
Analysis of evidence on knowledge of the risks of falling in hospitalized patients \\ Análisis de la evidencia sobre el conocimiento de los riesgos de caídas en pacientes hospitalizados
}

Recebido: 00/11/2021 | Revisado: 00/12/2021 | Aceito: 17/12/2021 | Publicado: 22/12/2021

\author{
Rhayssa Viegas Lima \\ ORCID: https://orcid.org/0000-0001-8987-1408 \\ Fundação Santa Casa de Misericórdia do Pará, Brasil \\ E-mail: rhay.maracema@ hotmail.com \\ Renato da Costa Teixeira \\ ORCID: https://orcid.org/0000-0002-4073-205X \\ Universidade Estadual do Pará, Brasil \\ E-mail: renatocteixeira@uepa.br \\ Thais de Oliveira Carvalho Granado Santos \\ ORCID: https://orcid.org/0000-0001-9496-4561 \\ Universidade Federal do Pará, Brasil \\ E-mail: thaissoliveira@yahoo.com.br \\ Ana Gabriela Sabaa Srur de Andrade \\ ORCID: https://orcid.org/0000-0002-5872-6950 \\ Fundação Santa Casa de Misericórdia do Pará, Brasil \\ E-mail: enfgabriela@hotmail.com \\ Xaene Maria Fernandes Duarte Mendonça \\ ORCID: https://orcid.org/0000-0002-0958-276X \\ Fundação Santa Casa de Misericórdia do Pará, Brasil \\ E-mail: xaenemaria@gmail.com \\ Pilar Maria de Oliveira Moraes \\ ORCID: https://orcid.org/0000-0003-2817-4574 \\ Fundação Santa Casa de Misericórdia do Pará, Brasil \\ E-mail: pilarmoraesnutri@gmail.com
}

\begin{abstract}
Resumo
Objetivo: analisar evidências científicas sobre o conhecimento dos riscos de queda em pacientes hospitalizados. Método: adotou-se como método a realização de uma revisão integrativa de literatura - RIL, para identificar e analisar as evidências sobre quedas em ambiente hospitalar. Foram utilizadas plataformas de pesquisa dentre elas Lilacs, Scielo, Pubmed e Google Acadêmico com as palavras "queda" "hospitalar" "acidente por queda" "queda hospitalar" "queda de paciente" foram combinadas tanto em português quanto em inglês. Foram incluídos artigos nestes dois idiomas, na íntegra, em um período que compreendeu os últimos 10 anos e que tinham como temática queda em ambiente hospitalar. Encontrou-se na busca 27 artigos e, após aplicados os critérios de exclusão, selecionou-se 12 artigos para leitura mais aprofundada. Conclusão: É importante observação e avaliação constante por parte da equipe de saúde quando se trata de quedas em especial quando o público envolvido é o idoso, podendo se valer de escalas tal qual a Morse FallScale.
\end{abstract}

Palavras-chave: Queda; Hospital; Acidente por queda; Queda hospitalar; Queda de paciente.

\begin{abstract}
Objective: to analyze scientific evidence on the knowledge of the risks of falling in hospitalized patients. Method: the method adopted was to carry out an integrative literature review - RIL, to identify and analyze evidence on falls in a hospital environment. Search platforms were used, including Lilacs, Scielo, Pubmed and Academic Google with the words "fall" "hospital" "accident by fall" "hospital fall" "patient fall" were combined both in Portuguese and in English. Articles in these two languages were included, in full, in a period that covered the last 10 years and which had the theme of falling in the hospital environment. The search found 27 articles and, after applying the exclusion criteria, 12 articles were selected for further reading. Conclusion: It is important to constantly observe and assess the health team when it comes to falls, especially when the public involved is the elderly, who can use scales such as the Morse FallScale.
\end{abstract}

Keywords: Fall; Hospital; Accident by fall; Hospital fall; Patient fall.

\section{Resumen}

Objetivo: analizar la evidencia científica sobre el conocimiento de los riesgos de caídas en pacientes hospitalizados. Método: el método adoptado fue realizar una revisión integradora de la literatura - RIL, para identificar y analizar evidencias sobre caídas en un ambiente hospitalario. Se utilizaron plataformas de búsqueda, incluidas Lilacs, Scielo, Pubmed y Academic Google con las palabras "caída" "hospital" "accidente por caída" "caída del hospital" "caída del 
paciente" se combinaron tanto en portugués como en inglés. Se incluyeron artículos en estos dos idiomas, en su totalidad, en un período que abarcó los últimos 10 años y cuya temática fue la caída en el ámbito hospitalario. La búsqueda encontró 27 artículos y, luego de aplicar los criterios de exclusión, se seleccionaron 12 artículos para lectura adicional. Conclusión: Es importante observar y evaluar constantemente al equipo de salud cuando se trata de caídas, especialmente cuando el público involucrado son las personas mayores, que pueden utilizar escalas como la Morse FallScale.

Palabras clave: Caída; Hospital; Accidente por caída; Caída del hospital; Caída del paciente.

\section{Introdução}

A segurança do paciente tem por definição a redução, a um mínimo aceitável, do risco de dano desnecessário associado ao cuidado em saúde (Silva-Batalha \& Melleiro, 2015; Brandão et al, 2018).

$\mathrm{Na}$ área hospitalar, tendo em vista a segurança do paciente, os chamados eventos adversos e sua prevenção são um dos alvos inerentes aos serviços de saúde. Definidos como danos ou lesões não intencionais, estes resultam em incapacidade ou disfunção de grande magnitude, comprometendo a saúde do indivíduo e prolongando sua permanência hospitalar, ainda que sob cuidados de uma equipe multiprofissional (Abreu et al, 2016).

Dentre os eventos adversos mais comuns estão as quedas de pacientes hospitalizados. Por definição, queda é um acontecimento involuntário, que leva o indivíduo inadvertidamente ao solo, podendo ser determinada por circunstâncias multifatoriais (Abreu et al, 2016; Bausch et al, 2017; Costa et al, 2019).

Tecnicamente é um deslocamento não intencional do corpo a um nível inferior, havendo incapacidade de correção em tempo hábil, com possibilidade de efeito danoso. Ainda que não haja encontro com o solo, caso o paciente seja encontrado no chão, em deslocamento ou necessite de amparo, tal situação também é considerada como "queda" (Silva, 2016; Santos et al, 2017; Vitorino et al, 2017; Luzia et al, 2018).

Os fatores de risco que predispõem a queda estão relacionados a diversas causas, classificadas em intrínsecas, as quais se relacionam a fatores fisiológicos do próprio paciente e que se dão através da diminuição da visão e audição, do dos distúrbios músculos esqueléticos, alterações de postura e equilíbrio (Borba et al, 2017; da Silva et al, 2017; Neves et al, 2017; Neves et al, 2016).

E causas extrínsecas, nas quais estão relacionadas ao ambiente no qual o indivíduo interage, estruturas físicas ou mudanças no referido ambiente, como má iluminação, tapetes e piso molhado. No caso de pacientes hospitalizados, há grande probabilidade de ocorrência de quedas devido ao ambiente desconhecido aliado ao quadro clínico desfavorável, ou seja, uma junção de ambos os fatores (da Silva et al, 2017; Neves et al, 2017; Neves et al, 2016; Vaccari et al, 2016; Luzia et al, 2016).

Cerca de $70 \%$ das ocorrências de queda acontecem no quarto do paciente, no momento das transferências do leito para cadeira ou cadeira de rodas e 19\% decorrentes de condições desfavoráveis para deambulação, o que evidencia um alto índice de quedas em ambiente hospitalar. Estes eventos complexos não só geram um agravamento no quadro do paciente, como aumentam seu tempo de internação, gerando custos sociais e emocionais e em casos extremos, a morte (Ebserh, 2017).

Considerando este agravo, as consequências são a nível burocrático para a equipe multiprofissional, já que haverá um aumento de custos hospitalares e uma perda de confiança nos profissionais e na instituição (Garcia, 2018;).

Considerando a assistência ao paciente, as ocorrências de quedas representam um indicador de qualidade do serviço prestado, o qual fornece embasamentos a equipe multiprofissional para planejar a melhor forma de oferecer o serviço. A preocupação em se evitar as quedas hospitalares promovem práticas mais seguras ainda que isso se apresente como um desafio para as instituições, que carecem de atitudes investigativas das causas de queda, bem como formulem e implementem medidas preventivas (Moreira, 2017; Cabral et al, 2016). Isto posto, este estudo teve por objetivo analisar evidências sobre o conhecimento dos riscos de queda em pacientes hospitalizados. 


\section{Metodologia}

Para responder ao objetivo do estudo, adotou-se como método a realização de uma revisão integrativa de literatura RIL, para identificar e analisar as evidências sobre quedas em ambiente hospitalar.

A RIL é tida como um método de pesquisa que possibilita uma síntese de conhecimentos através de processo sistemático e rigoroso. Por ser fundamentada em conhecimento científico, permite incorporação de evidências na prática clínica com resultados de qualidade. A condução de uma RI requere um rigor metodológico em suas fases tal qual ocorre no desenvolvimento de pesquisas, tais fases são: elaboração das questões da revisão, busca e seleção dos estudos na literatura, extração de dados dos estudos, avaliação dos dados contidos nos estudos incluídos na revisão, síntese dos resultados da revisão e apresentação da revisão (de Sousa, 2017; Mendes, 2019).

A partir do conceito de quedas, definiram-se as questões: qual o conceito de queda? Como esta se mostra no meio hospitalar? O quanto se sabe sobre os riscos para a ocorrência da queda em hospitais? Para responder as questões norteadoras, foram utilizadas plataformas de pesquisa dentre elas Lilacs, Scielo, Pubmed e Google Acadêmico. Ao se realizar a pesquisa, as palavras "queda" "hospitalar" "acidente por queda" "queda hospitalar" "queda de paciente" foram combinadas tanto em português quanto em inglês. Foram incluídos artigos nestes dois idiomas, na íntegra, em um período que compreende os últimos 10 anos e que tivessem como temática queda em ambiente hospitalar.

Como critérios de exclusão, não foram utilizados resumos, artigos com temática de queda em ambiente doméstico ou outro que não fosse o hospitalar e cujo público não fosse o adulto. Artigos cuja metodologia implicasse em alguma intervenção científica ou aplicação de teste relacionado a queda também foram excluídos. Definidos os critérios de exclusão, foi realizada coleta de artigos nas referidas plataformas citadas, da qual 27 artigos foram eleitos para uma pré-seleção. Dos artigos préselecionados, 12 foram elencados para leitura aprofundada, análise de metodologia e resultados e fichamento das partes mais importantes. Posteriormente, realizou-se construção de parte escrita do artigo, conforme apresentado em Fluxograma 1. 
Fluxograma 1: Processo de produção da Revisão Integrativa de Literatura sobre quedas hospitalares de publicação de 2011 a 2021, Belém- Pará.
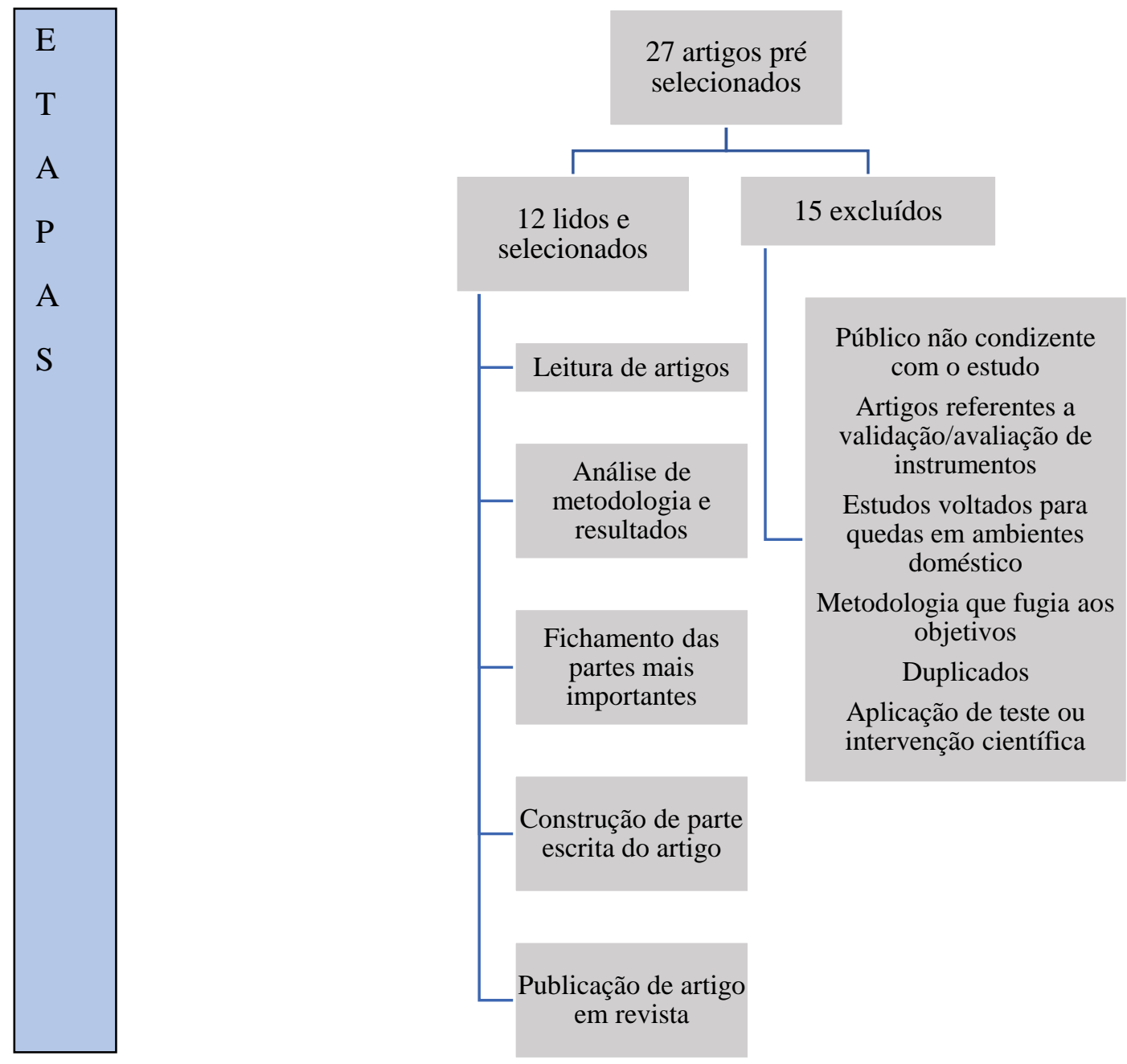

Fonte: Autores (2021).

\section{Resultados}

Encontrou-se na busca 27 artigos para pré-seleção e, após aplicados os critérios de exclusão, selecionou-se 12 artigos para leitura mais aprofundada. Os artigos excluídos não atendiam a metodologia deste estudo por possuir objetivos relacionados a abordagem de equipe de enfermagem ao invés de pacientes, validação de instrumentos e escalas ou se tratavam de quedas em ambiente doméstico.

Já com relação aos artigos selecionados para esta revisão, 9 foram escritos em língua nativa, 2 em inglês e 1 em espanhol. As informaç̃̃es com relação aos autores, periódicos, ano de publicação, métodos e resultados estão agrupadas nos quadros 1 e 2 a seguir. 
Quadro 1 - Artigos selecionados sobre quedas hospitalares publicados no período de 2011 a 2021, Belém- Pará.

\begin{tabular}{|c|c|c|c|c|}
\hline Autores & Título & Periódico & Tipo de estudo & Ano \\
\hline da Silva Pereira et al. & $\begin{array}{l}\text { Quedas em pessoas idosas hospitalizadas: uma revisão } \\
\text { integrativa }\end{array}$ & $\begin{array}{l}\text { Revista Enfermagem } \\
\text { Atual }\end{array}$ & $\begin{array}{l}\text { Revisão Integrativa } \\
\text { de Literatura }\end{array}$ & 2018 \\
\hline Cassola et al. & $\begin{array}{l}\text { Quedas em pacientes psiquiátricos: uma revisão } \\
\text { integrativa }\end{array}$ & Ciência, cuidado e saúde & $\begin{array}{c}\text { Revisão Integrativa } \\
\text { de Literatura }\end{array}$ & 2019 \\
\hline Luzia et al. & $\begin{array}{l}\text { Características das quedas com dano em pacientes } \\
\text { hospitalizados }\end{array}$ & $\begin{array}{l}\text { Revista Gaúcha de } \\
\text { Enfermagem }\end{array}$ & $\begin{array}{l}\text { Estudo longitudinal, } \\
\text { retrospectivo }\end{array}$ & 2019 \\
\hline Vitor \& de Araújo & $\begin{array}{l}\text { Definições para o resultado de enfermagem } \\
\text { comportamento de prevenção de quedas: uma revisão } \\
\text { integrativa }\end{array}$ & $\begin{array}{l}\text { Revista Eletrônica de } \\
\text { Enfermagem }\end{array}$ & $\begin{array}{l}\text { Revisão Integrativa } \\
\text { de Literatura }\end{array}$ & 2011 \\
\hline Barbosa et al. & $\begin{array}{l}\text { Caracterização dos incidentes de quedas de pacientes } \\
\text { adultos internados em um hospital universitário }\end{array}$ & $\begin{array}{l}\text { Revista Gaúcha de } \\
\text { Enfermagem }\end{array}$ & $\begin{array}{l}\text { Estudo descritivo, } \\
\text { transversal e } \\
\text { retrospectivo }\end{array}$ & 2019 \\
\hline Kobayashi et al. & Characteristics of out patient falls that occurred in hospital & Nagoya J. Med. Sci. & $\begin{array}{l}\text { Estudo descritivo, } \\
\text { retrospectivo }\end{array}$ & 2018 \\
\hline Leiva et al. & $\begin{array}{l}\text { Factores asociados a caídas en adultos mayores chilenos: } \\
\text { evidencia de La Encuesta Nacional de Salud 2009-2010 }\end{array}$ & $\begin{array}{l}\text { Revista de Medicina do } \\
\text { Chile }\end{array}$ & $\begin{array}{l}\text { Estudo analítico, } \\
\text { observacional }\end{array}$ & 2019 \\
\hline da Rosa et al. & $\begin{array}{c}\text { Avaliação do risco de quedas de pacientes em serviço de } \\
\text { emergência }\end{array}$ & $\begin{array}{l}\text { Revista de Enfermagem } \\
\text { do Centro-Oeste Mineiro }\end{array}$ & $\begin{array}{l}\text { Estudo descritivo, } \\
\text { quantitativo, } \\
\text { transversal }\end{array}$ & 2019 \\
\hline Remor et al. & $\begin{array}{l}\text { Análise dos fatores de risco para queda de adultos nas } \\
\text { primeiras } 48 \text { horas de hospitalização }\end{array}$ & $\begin{array}{l}\text { Revista Gaúcha de } \\
\text { Enfermagem }\end{array}$ & $\begin{array}{l}\text { Estudo de coorte } \\
\text { prospectivo }\end{array}$ & 2014 \\
\hline de Araújo Sarges et al. & $\begin{array}{c}\text { Avaliação da segurança do idoso hospitalizado quanto ao } \\
\text { risco de quedas }\end{array}$ & $\begin{array}{l}\text { Revista Brasileira de } \\
\text { Enfermagem }\end{array}$ & $\begin{array}{c}\text { Estudo } \\
\text { epidemiológico, } \\
\text { transversal, } \\
\text { prospectivo, } \\
\text { descritivo }\end{array}$ & 2017 \\
\hline Bittencourt et al. & $\begin{array}{l}\text { Fatores associados ao risco de quedas em pacientes } \\
\text { adultos hospitalizados }\end{array}$ & $\begin{array}{l}\text { Revista da Escola de } \\
\text { Enfermagem da USP }\end{array}$ & $\begin{array}{l}\text { Estudo transversal, } \\
\text { analítico e } \\
\text { quantitativo }\end{array}$ & 2017 \\
\hline Sato et al. & $\begin{array}{c}\text { Falls among Hospitalized Patients in na Acute Care } \\
\text { Hospital: Analyses of Incident Reports }\end{array}$ & $\begin{array}{l}\text { The Journal of Medical } \\
\text { Investigation }\end{array}$ & $\begin{array}{l}\text { Estudo descritivo, } \\
\text { retrospectivo }\end{array}$ & 2018 \\
\hline
\end{tabular}

Fonte: Autores (2021).

Quadro 2 - Objetivo, Método e Resultados dos artigos selecionados para artigos selecionados sobre quedas hospitalares publicados no período de 2011 a 2021, Belém- Pará.

\begin{tabular}{|c|c|c|c|}
\hline Autores & Objetivo & Método & Resultados \\
\hline $\begin{array}{l}\text { Pereira et } \\
\text { al. }\end{array}$ & $\begin{array}{l}\text { Analisar as evidências } \\
\text { científicas acerca das quedas de } \\
\text { idosos em ambiente hospitalar }\end{array}$ & $\begin{array}{l}\text { Realizada revisão integrativa da literatura acerca das } \\
\text { publicações relacionadas à queda em idosos } \\
\text { hospitalizados no período de } 2011 \text { a } 2015 \text {. }\end{array}$ & $\begin{array}{l}\text { Foram selecionados sete artigos para revisão que } \\
\text { demonstraram que os fatores de risco para quedas em } \\
\text { idosos hospitalizados estavam diretamente ligados a } \\
\text { acuidade visual, mobilidade física, alterações } \\
\text { cognitivas e incontinência urinária. }\end{array}$ \\
\hline $\begin{array}{l}\text { Cassola } \\
\text { et al. }\end{array}$ & $\begin{array}{l}\text { Caracterizar a produção sobre o } \\
\text { evento quedas em pacientes } \\
\text { psiquiátricos adultos. }\end{array}$ & $\begin{array}{l}\text { Tratou-se de uma revisão integrativa que utilizou os } \\
\text { pressupostos teórico-metodológicos propostos por } \\
\text { Whittemore }\end{array}$ & $\begin{array}{l}\text { A partir do percurso analítico, foi obtido um total de } \\
57 \text { artigos, divididos em estudos quantitativos, } \\
\text { qualitativos e de revisão. Os cenários que mais se } \\
\text { destacaram para ocorrência de queda foram centros } \\
\text { comunitários e instituições hospitalares. Dos } 57 \\
\text { artigos, } 25 \text { se preocupavam com identificação dos } \\
\text { fatores de queda. }\end{array}$ \\
\hline $\begin{array}{l}\text { Luzia et } \\
\text { al. }\end{array}$ & $\begin{array}{l}\text { Descrever as características das } \\
\text { quedas com dano de pacientes, } \\
\text { seus fatores de risco e lesões } \\
\text { decorrentes. }\end{array}$ & $\begin{array}{l}\text { Foi realizada análise de } 260 \text { registros de pacientes que } \\
\text { sofreram quedas com dano notificadas no período de } \\
\text { setembro de } 2012 \text { a junho de } 2017 \text {. Para o dano fora } \\
\text { adotada classificação entre leve, moderado e grave. }\end{array}$ & $\begin{array}{l}\text { No período foram notificadas } 597 \text { quedas, em } 260 \\
(43 \%) \text { foi evidenciado dano relacionado ao evento. Os } \\
\text { pacientes que apresentaram queda com dano eram na } \\
\text { sua maioria idosos }(78 \%) \text {, do sexo feminino }(55 \%) \text {, em } \\
\text { tratamento clínico }(68 \%) \text { e desacompanhados }(59,4 \%) \text {. } \\
\text { As quedas ocorreram da própria altura }(63,4 \%) \text { e no } \\
\text { quarto do paciente }(67,3 \%) \text {. A gravidade dos danos foi } \\
\text { leve em } 80,8 \% \text { dos casos, grave em } 11,9 \% \text { e moderado, } \\
7,3 \% \text {. Foram identificados cinco óbitos decorrentes de } \\
\text { quedas }(1,9 \%) \text {. }\end{array}$ \\
\hline $\begin{array}{l}\text { Vitor \& } \\
\text { de Araújo }\end{array}$ & $\begin{array}{l}\text { Identificar evidências na } \\
\text { literatura sobre as medidas de } \\
\text { prevenção de quedas e os fatores } \\
\text { de risco para quedas em }\end{array}$ & $\begin{array}{l}\text { Foi utilizada revisão integrativa como ferramenta para } \\
\text { a identificação e análise de evidência que sustentassem } \\
\text { a revisão do resultado de enfermagem NOC } \\
\text { Comportamento para Prevenção de Quedas. Para isso, }\end{array}$ & $\begin{array}{l}\text { Quanto à avaliação dos estudos selecionados, } 42 \\
\text { respondiam às questões norteadoras. Os indicadores } \\
\text { Uso de recursos de correção da visão e Uso de sapatos } \\
\text { amarrados e do tamanho adequado foram considerados }\end{array}$ \\
\hline
\end{tabular}




\begin{tabular}{|c|c|c|c|}
\hline & $\begin{array}{llr}\text { pacientes } & \text { internados } \quad \text { em } \\
\text { ambiente e } & \text { compará-las com } \\
\text { indicadores } & \text { NOC (Nursing Out } \\
\text { comes Classification) existentes }\end{array}$ & $\begin{array}{l}\text { foram selecionadas as bases de dados Cinahl, Pubmed, } \\
\text { Lilacs e Cochrane e o Dedalus }\end{array}$ & $\begin{array}{l}\text { insuficientes para avaliar fatores de risco como déficits } \\
\text { sensoriais e roupas/calçados inadequados. }\end{array}$ \\
\hline $\begin{array}{l}\text { Barbosa } \\
\text { et al. }\end{array}$ & $\begin{array}{l}\text { Avaliar as notificações e } \\
\text { caracterizar os incidentes de } \\
\text { quedas dos pacientes adultos } \\
\text { internados em unidades clínicas } \\
\text { e cirúrgicas de um hospital } \\
\text { universitário na região sul do } \\
\text { país, no período de } 2011 \text { a } 2014\end{array}$ & $\begin{array}{l}\text { Estudo descritivo, transversal e retrospectivo, } \\
\text { realizado no período de dezembro de } 2016 \text { a dezembro } \\
\text { de 2017. A amostra foi de } 1112 \text { notificações, as quais } \\
\text { abrangiam os pacientes internados com notificações de } \\
\text { ocorrência de quedas }\end{array}$ & $\begin{array}{l}\text { A taxa de incidência de quedas no período estudado foi } \\
\text { de } 1,61 \text { quedas/1000 pacientes/dia em } 2011,2,03 \mathrm{em} \\
2012,1,83 \mathrm{em} 2013 \text { e } 1,62 \mathrm{em} 2014 \text {. Foram } \\
\text { predominantes na amostra os pacientes do sexo } \\
\text { feminino e idosos, onde } 69,4 \% \text { dos incidentes não } \\
\text { apresentaram dano. A ocorrência de quedas foi } \\
\text { significativamente maior no período noturno. } \\
\text { Limitação para deambular e estar desacompanhado } \\
\text { foram os fatores mais prevalentes nas condições do } \\
\text { paciente antes da queda. }\end{array}$ \\
\hline $\begin{array}{l}\text { Kobayas } \\
\text { hi et al. }\end{array}$ & $\begin{array}{l}\text { Relatar as características das } \\
\text { quedas ambulatoriais que } \\
\text { ocorreram no hospital durante } \\
\text { cinco anos para identificar } \\
\text { fatores associados à queda } \\
\text { nesses pacientes. }\end{array}$ & $\begin{array}{l}\text { Foram investigados incidentes de quedas } \\
\text { ambulatoriais no período de abril/2012 a março/2017 } \\
\text { através de um banco de dados no sistema de relatórios } \\
\text { de eventos hospitalares. }\end{array}$ & $\begin{array}{l}\text { Um total de } 3758 \text { pacientes caíram no hospital, } \\
\text { incluindo um quantitativo de } 146 \text { pacientes } \\
\text { ambulatoriais. Maior parte das quedas ocorreu com } \\
\text { pessoas idosas (acima de } 70 \text { anos) no próprio quarto, } \\
\text { seguido de salas de exame, escadas rolantes e salas de } \\
\text { espera. A maior parte das quedas ocorreu em pacientes } \\
\text { neurológicos, neurocirúrgicos e oftalmológicos, dentre } \\
\text { os danos decorrentes de quedas destacaram-se } 4 \\
\text { fraturas de fêmur, uma lesão dentária e uma fratura de } \\
\text { púbis. }\end{array}$ \\
\hline $\begin{array}{l}\text { Leiva } \\
\text { Cigarroa- } \\
\text { Cuevas et } \\
\text { al. }\end{array}$ & $\begin{array}{l}\text { Investigar os fatores associada a } \\
\text { quedas em idosos chilenos }\end{array}$ & $\begin{array}{l}\text { Este estudo incluiu } 1.334 \text { participantes mais de } 60 \text { anos } \\
\text { da Pesquisa Nacional de Saúde do Chile (ENS 2009- } \\
\text { 2010). Foram analisados os auto relatos destes } \\
\text { indivíduos com relação a ocorrência de quedas, estilo } \\
\text { de vida, status sócio-demográfico e de saúde. }\end{array}$ & $\begin{array}{l}\text { As quedas foram relatadas por } 37 \% \text { dos participantes, } \\
\text { a frequência maior se deu em mulheres e aqueles com } \\
\text { idade superior a } 70 \% \text {. Os fatores relacionados a queda } \\
\text { mais citados foram deficiência auditiva visão } \\
\text { prejudicada, pouco relato de bem-estar, e } \\
\text { incapacidades. }\end{array}$ \\
\hline $\begin{array}{l}\text { da Rosa } \\
\text { et al. }\end{array}$ & $\begin{array}{l}\text { Identificar o risco de quedas nos } \\
\text { pacientes em observação em um } \\
\text { serviço de emergência, por meio } \\
\text { da aplicação da Escala }\end{array}$ & $\begin{array}{l}\text { Estudo quantitativo, com dados transversais do tipo } \\
\text { descritivo, desenvolvido com } 30 \text { pacientes internados } \\
\text { em um serviço de emergência do Rio Grande do Sul. } \\
\text { Os dados foram coletados, entre junho e julho/2018, } \\
\text { pela aplicação da Escala de Quedas de Morse. }\end{array}$ & $\begin{array}{l}\text { Dos } 30 \text { pacientes, } 15 \text { eram do sexo feminino e } 15 \text { do } \\
\text { masculino, com idade entre } 30 \text { e } 83 \text { anos. Destes, } 16 \\
(53,33 \%) \text { apresentaram alto, } 07(23,33 \%) \text { médio e } 07 \\
(23,33 \%) \text { baixo risco para quedas. Os fatores de risco } \\
\text { mais encontrados foram: terapia endovenosa, } \\
\text { diagnóstico secundário e marcha. }\end{array}$ \\
\hline $\begin{array}{l}\text { Remor et } \\
\text { al. }\end{array}$ & $\begin{array}{l}\text { Analisar os fatores de risco para } \\
\text { quedas nas primeiras } 48 \text { horas } \\
\text { de hospitalização e associá-los } \\
\text { com a ocorrência de quedas }\end{array}$ & $\begin{array}{l}\text { Estudo de coorte, realizado em um hospital de Porto } \\
\text { Alegre/RS. A população do estudo foram os pacientes } \\
\text { adultos hospitalizados, e a amostra configurou-se em } \\
556 \text { pacientes avaliados quanto aos fatores associados } \\
\text { a quedas em até } 48 \text { horas do início da internação, como } \\
\text { a Morse Fall Scale e a forca muscular, dentre outros. }\end{array}$ & $\begin{array}{l}\text { Através da classificação pela Morse Fall Scale, foi } \\
\text { notificado risco elevado de quedas. Os itens } \\
\text { específicos de história de quedas, auxilio na } \\
\text { deambulação, marcha comprometida/cambaleante } \\
\text { Foram associados com as quedas durante } \\
\text { hospitalização. Além desses fatores de risco, a forca } \\
\text { muscular reduzida e o déficit visual contribuíram para } \\
\text { a ocorrência desse incidente de segurança }\end{array}$ \\
\hline $\begin{array}{l}\text { de Araújo } \\
\text { Sarges et } \\
\text { al. }\end{array}$ & $\begin{array}{l}\text { Avaliação da segurança do } \\
\text { idoso hospitalizado quanto ao } \\
\text { risco de quedas Avaliar a } \\
\text { segurança do idoso } \\
\text { hospitalizado quanto ao risco } \\
\text { para quedas segundo os } \\
\text { parâmetros da Morse Fall Scale }\end{array}$ & $\begin{array}{l}\text { Estudo de cunho epidemiológico, transversal, } \\
\text { prospectivo e descritivo, realizado em um hospital } \\
\text { público em Belém. Foram selecionados } 71 \text { idosos que } \\
\text { estiveram hospitalizados por mais de } 72 \text { horas. A esta } \\
\text { amostra foi aplicada dois protocolos: um com } \\
\text { informações sociodemográficas e outro foi a Morse } \\
\text { Fall Scale para avaliar o risco de quedas. }\end{array}$ & $\begin{array}{l}\text { A média de idade encontrada foi de } 71,3 \text { anos; } 58,7 \% \\
\text { do sexo masculino; } 44 \% \text { com baixa escolaridade; } \\
38,7 \% \text { internados por doenças cardiovasculares. } \\
\text { Houve risco elevado para quedas em } 52 \% \text { dos idosos. }\end{array}$ \\
\hline $\begin{array}{l}\text { Bittencou } \\
\text { rt et al. }\end{array}$ & $\begin{array}{l}\text { Analisar os fatores relacionados } \\
\text { ao risco de quedas em pacientes } \\
\text { adultos internados em um } \\
\text { hospital }\end{array}$ & $\begin{array}{l}\text { Estudo quantitativo, transversal e analítico realizado } \\
\text { com } 612 \text { pacientes, os quais foram submetidos a dois } \\
\text { instrumentos: um protocolo contendo questões sobre } \\
\text { situação sociodemográfica e clínica e o outro sendo a } \\
\text { Morse Fall Scale. }\end{array}$ & $\begin{array}{l}\text { Dentre os pacientes, } 62,3 \% \text { do sexo feminino; } 54,6 \% \\
\text { dos pacientes com idade menor que } 60 \text { anos. Quanto } \\
\text { ao risco de queda, de acordo com a Escala de Morse, } \\
38,2 \% \text { dos pacientes foram classificados com risco } \\
\text { baixo de queda, } 30,1 \% \text { com risco moderado e } 31,7 \% \\
\text { com risco elevado. Foi encontrada uma associação } \\
\text { entre o alto risco de quedas e hospitalização } \\
\text { neurológica clínica, trauma cirúrgico (hospitalização) } \\
\text { e presença de comorbidades. }\end{array}$ \\
\hline Sato e & $\begin{array}{l}\text { Investigar a incidência e as } \\
\text { características de quedas entre } \\
\text { pacientes hospitalizados em um } \\
\text { hospital de cuidados intensivos } \\
\text { a partir de relatórios de } \\
\text { incidentes por funcionários } \\
\text { entre janeiro e junho de } 2013 \text {. }\end{array}$ & $\begin{array}{l}\text { Este estudo foi realizado no Tokushima University } \\
\text { Hospital, através de avaliação retrospectiva de quedas, } \\
\text { por meio de relatórios de incidentes e registros } \\
\text { médicos dos pacientes hospitalizados, durante seis } \\
\text { meses, de janeiro a junho de } 2013 \text {. }\end{array}$ & $\begin{array}{l}\text { Em } 135 \text { pacientes, houve um quantitativo de } 154 \\
\text { quedas em } 135 \text { pacientes, destes eventos, dois } \\
\text { resultaram em fratura. A maior parte dos pacientes que } \\
\text { tiveram queda possuía idade por volta de } 63,9 \text { anos. } \\
\text { Muitas destas quedas ocorreram à beira do leito } \\
(68,2 \%) \text {. O departamento de medicina respiratória e } \\
\text { reumatologia teve a maior taxa de queda seguida pelos } \\
\text { departamentos de neurocirurgia e neurologia. }\end{array}$ \\
\hline
\end{tabular}

Fonte: Autores (2021). 


\section{Discussão}

A queda é o evento no qual um indivíduo vem a permanecer no solo ou em outro nível inferior, descartando mudanças de posição intencionais seja para se apoiar em mobílias, paredes ou quaisquer outros objetos (Luzia et al, 2019).

Quando se trata de pacientes hospitalizados, as consequências de uma queda são quase imediatas. Além de danos teciduais, como fraturas e traumas que afetam diretamente as atividades de vida diária do paciente, há influência no aumento do tempo de internação, custos elevados no tratamento e descrença do paciente com relação a equipe de trabalho (da Rosa et al, 2019; Kobayashi et al, 2018).

A redução de danos desnecessários ao paciente é o ponto básico da chamada segurança do paciente. Tal redução deve se dar pela assistência até um nível mínimo aceitável nas instituições hospitalares. Muitas discussões tem sido geradas assim como alertas na área da saúde visando diminuir atos assistenciais inseguros de modo que os pacientes tenham acesso a um melhor serviço (Remor et al, 2014).

Um estudo analisou os fatores de risco nas primeiras $48 \mathrm{~h}$ de internação hospitalar, analisando sua relação com as ocorrências de queda nos pacientes hospitalizados. Uma das etapas do referido estudo incluía avaliação de força muscular e avaliação dos riscos de queda através da Morse FallScale traduzida (Remor et al, 2014).

Dos 556 pacientes, 44 (79\%) apresentaram quedas, a maioria possuía idade superior a 58 anos, o que a caracteriza como população idosa e eram do sexo masculino. Dos fatores evidentes que estavam associados com as quedas destes pacientes estão o déficit visual, reduzida força muscular e classificação elevada para o risco de quedas (Remor et al, 2014).

As quedas que ocorrem durante o período de internação são consideradas significativos eventos de quebras de segurança do paciente durante a internação, frequentemente responsáveis pelo aumento do número de dias de hospitalização com piores condições na recuperação (Sarges et al, 2017).

A avaliação do paciente com relação ao risco de quedas, tanto no momento de sua admissão quanto no decorrer de sua internação mostra-se importante, já que a partir dela se tem uma importante ferramenta de cuidado por parte da equipe de saúde. Outro estudo realizado no âmbito hospitalar em serviços de emergência também evidenciou alto risco para a ocorrência de quedas através da Morse FallScale. Dos 30 pacientes participantes do estudo, 16 (53,33\%) apresentaram alto risco para quedas em contrapartida de $07(23,33 \%)$ com médio risco e $07(23,33 \%)$ com baixo risco. Outra similaridade diz respeito a faixa etária, $56,67 \%$ dos pacientes possuíam idade maior de 60 anos (da Rosa et al, 2019).

O ambiente de uma emergência hospitalar traz consigo características físicas muito específicas como corredores abarrotados, reduzido espaço para locomoção associado com alto fluxo pessoal, aumentando risco de queda, permanência dos pacientes nestes serviços por longos períodos, o que exige maior complexidade da assistência. Dessa forma, os serviços emergenciais atribuem alto risco de queda para os pacientes que fazem uso dele por apresentarem inadequações estruturais (da Rosa et al, 2019).

A utilização da Escala de Morse tem se mostrado eficaz e amplamente utilizada em vários outros estudos, por possuir alta sensibilidade na classificação do risco de queda. Um estudo realizado em um Hospital Privado do Rio Grande do Sul abordou as quedas tidas por previsíveis e detectáveis através da já citada Morse FallScale. Dos 612 pacientes, ao contrário dos artigos supracitados, a maior parte da amostra era constituída por indivíduos com idade abaixo dos 60 anos (54,6\%) (Bittencourt et al, 2017).

Com relação ao risco de queda, de acordo com a escala, $38,2 \%$ foram classificados com baixo risco, contudo verificouse associação significativa entre a idade, pacientes idosos e não-idosos, com o rico de cair; os pacientes que possuíam internação clínica apresentavam alto risco de quedas segundo a Morse FallScale. Entre os pacientes clínicos com risco elevado de queda, a maioria foi de internação neurológica (59,4\%), seguida de internação respiratória (48,1\%) (Bittencourt et al, 2017). 
Em outro hospital, também da região Sul, foram analisados 260 registros de pacientes que sofreram quedas que resultaram em danos. Notificaram-se 597 quedas, evidenciando uma incidência anual de 1,3 a 2,5 quedas para mil pacientes/dia. Do total, em $43 \%$ do total de quedas, houve evidência de danos relacionados ao evento, sendo a maior parte deles de cunho leve. Dentre os fatores de risco mais evidentes, estavam inclusos idade acima de 65 anos, alteração de nível de consciência, afecção da marcha ou prejuízo físico, alteração da visão ou uso de medicamentos de risco (Luzia et al, 2019).

Danos resultantes de quedas corroboram com os resultados de um estudo que objetivou relatar as características das quedas ambulatoriais. De 3758 pacientes, 146 pacientes de cunho ambulatorial apresentaram quedas. A faixa etária predominante era de 70 anos e a ocorrência em sua maioria foi em salas de cirurgia, sendo citadas também salas de exame, escadas rolantes e salas de espera. Foram notificados danos principais resultantes das quedas, dentre os quais o que se sobressaiu foi a fratura de colo de fêmur (Kobayashi et al, 2018).

Assim como em ambiente ambulatorial, pesquisa realizada em um hospital de cuidados intensivos também notificou quedas resultantes em danos para o paciente. Foi realizada uma investigação retrospectiva sobre as características e incidência de quedas no referido hospital, das 154 quedas, duas resultaram em fraturas e corroborando com a maior parte dos artigos já citados, a idade média dos pacientes foi de 63,9 anos, acontecendo em sua maioria, à beira leito (Sato et al, 2018).

Tem sido aferido que dentre os fatores de risco para quedas, há um predomínio de citações sobre déficits sensoriais, condições ambientais que favorecem a ocorrência de queda, mobilidade, deambulação afetada, marcha ou flexibilidade alteradas, problemas de equilíbrio, condições médicas especiais que dão maior propensão ou idade avançada. Uma vez que tais fatores são compreendidos, as quedas assumem uma posição de evento multifatorial, sujeito a variar conforme variam seus fatores de risco (Vitor \& de Araújo, 2011; Cassola et al, 2019).

Considerando as internações hospitalares e o quanto podem ocasionar risco de queda e o próprio evento da mesma, há uma necessidade de atenção por parte da equipe de saúde. Neste âmbito, em especial a população idosa tem considerada diminuição de sua capacidade funcional. Aliando a fragilidade física, vulnerabilidade emocional e internações o processo de saúde e doença se potencializa em riscos para ocorrência (da Silva Pereira et al, 2018).

Os idosos tendem a serem os maiores consumidores dos serviços de saúde, com internações mais frequentes que as outras faixas etárias. Os acometimentos são múltiplos de natureza crônica, exigindo um acompanhamento médico e multidisciplinar mais atencioso e considerando o fator queda, estas não se distribuem de forma uniforme, corroborando com boa parte da literatura, as ocorrências se concentram em pacientes idosos, neurológicos e em reabilitação (Sarges et al, 2017).

Um estudo realizado em um hospital universitário do Sul caracterizou os incidentes de quedas dos pacientes adultos e foram predominantes na amostra pacientes do sexo feminino e idosos, os incidentes, todavia, não geraram dano. Alguns fatores como limitação para deambular e estar desacompanhado prevaleceram dentre as condições que propiciaram a queda (Barbosa et al, 2019).

Os referidos resultados combinam com os encontrados em uma pesquisa que investigou os fatores associados a quedas em idosos chilenos. Os autores analisaram os autorrelatos de quedas de 1334 pessoas com idade acima de 60 anos, sendo que foram registradas ocorrências de quedas nos últimos 12 meses em cerca de 37\% dos entrevistados. A prevalência foi maior em mulheres e fatores intrínsecos como deficiência auditiva, visão prejudicada e deficiência foram citados (Leiva et al, 2019).

\section{Conclusão}

Este estudo evidenciou que a queda é um evento adverso, todavia evitável em sua grande maioria a partir da identificação de fatores de risco (extrínsecos e intrínsecos) e medidas de mitigação dos mesmos, principalmente no âmbito da assistência em saúde. 
A ocorrência do evento no ambiente hospitalar tende a promover danos imediatos, ocasionando ônus para o serviço, prolongamento de tempo de internação e perda de confiança para com a equipe cuidadora. Logo se faz necessária vigilância constante por parte da equipe de saúde, especialmente em setores nos quais prevalecem pacientes com faixa etária acima dos 60 anos (população idosa) que possui maiores fatores de risco à queda. As análises das publicações demonstraram a importância do uso da escala de Morse FallScale para avaliação de riscos intrínsecos e extrínsecos como modo de prevenir quedas.

Ainda que seja um assunto de relevância e pertinência para a área da saúde, se fazem necessários mais estudos com abordagem focada no conhecimento e percepção sobre os riscos de quedas relacionados a pacientes no âmbito hospitalar, de modo que se possa promover futuras ações que minimizem a ocorrência do evento adverso.

\section{Referências}

Abreu, D. R. D. O. M., de Oliveira, J. L. C., de Abreu, A. R. G., \& Abreu, H. C. A. (2016). Quedas no ambiente hospitalar, qualidade e segurança do paciente: metassíntese da literatura. Revista Eletrônica Gestão e Saúde, (3), 1244-1255.

Barbosa, A. D. S., Chaves, E. H. B., Ribeiro, R. G., Quadros, D. V. D., Suzuki, L. M., \& Magalhães, A. M. M. D. (2019). Caracterização dos incidentes de quedas de pacientes adultos internados em um hospital universitário. Revista gaucha de enfermagem, 40.

Bausch, A. B., Waterkemper, R., da Costa Linch, G. F., Paz, A. A., \& Pelegrini, A. H. W. (2017). Mortalidade por quedas de leitos hospitalares: estudo retrospectivo. Revista Baiana de Enfermagem 31 ,(2).

Bittencourt, V. L. L., Graube, S. L., Stumm, E. M. F., Battisti, I. D. E., Loro, M. M., \& Winkelmann, E. R. (2017). Fatores associados ao risco de quedas em pacientes adultos hospitalizados. Revista da Escola de Enfermagem da USP, 51.

Borba, D. L. L., Lange, C., Llano, P. M. P., Maagh, S. B., da Costa Viegas, A., \& dos Santos, B. P. (2017). Perfil das idosas atendidas por queda em um serviço de emergência. Journal of Nursing and Health, 7(1), 67-77.

Brandão, M. G. S. A., Brito, O. D., \& Barros, L. M. (2018). Gestão de riscos e segurança do paciente: mapeamento dos riscos de eventos adversos na emergência de um hospital de ensino. Revista de Administração em Saúde, 18(70).

Cabral, J. V. B., de Souza Silva, C. M., Bispo, D. J. S., \& Silva, E. M. (2016). Fatores de risco para quedas em idosos hospitalizados. HOLOS, 3 , $328-337$.

Cassola, T. P., Eslabão, A. D., dos Santos, E. O., Cruz, I. R., Schneider, J. F., \& de Pinho, L. B. (2019). < b> Quedas em pacientes psiquiátricos: uma revisão integrativa/Psychiatric patients' falls: an integrative review. Ciência, Cuidado e Saúde, 18(3).

Costa, J. C., da Silva Junqueira, M., da Silva Gomes, M. M., \& Nova, J. C. V. (2019). Importância da equipe multiprofissional na prevenção de quedas em idosos no ambiente hospitalar. REMAS-Revista Educação, Meio Ambiente e Saúde, 9(3), 89-106.

da Rosa, P. H., Rangel, R. F., Machado, K. D. F. C., Cesar, M. P., \& Ilha, S. (2019). Avaliação do risco de quedas de pacientes em serviço de emergência. Revista de Enfermagem do Centro-Oeste Mineiro, 9.

da Silva Pereira, E., Sá, S. P. C., de Oliveira Ferreira, A. M., Lago, E. L. M., Pimentel, D. F., \& da Conceição Oliveira Filho, V. (2018). Quedas em pessoas idosas hospitalizadas: uma revisão integrativa. Revista Enfermagem Atual In Derme, 85(23).

da Silva, F. A., de Matos, M. I. B., \& Esteves, L. S. F. (2017, August). Avaliação do risco de queda em idosos independentes. In Colloquium Vitae. ISSN: 19846436 (Vol. 9, No. 1, pp. 18-22).

de Sousa, L. M. M., Marques-Vieira, C. M. A., Severino, S. S. P., \& Antunes, A. V. (2017). A metodologia de revisão integrativa da literatura em enfermagem. $N^{o} 21$ Série 2-Novembro 2017, 17.

Ebserh (2017). Protocolo de prevenção de queda. Disponível a partir de: http://ebserh.gov.br/documents/214336/1110036/PRO.NUSEP.003++PROTOCOLO+DE+PREVEN\%C3\%87\%C3\%83O+DE+QUEDAS.pdf/12e03eba-94ad-40f5-a3f3-837127a73431

Garcia, D. D. C. (2018). Segurança do paciente: análise de eventos adversos relacionados às quedas de pacientes hospitalizados.. Dissertação [Mestrado em Enfermagem] - Universidade Estadual Paulista "Júlio de Mesquita Filho"

Kobayashi, K., Ando, K., Suzuki, Y., Inagaki, Y., Nagao, Y., Ishiguro, N., \& Imagama, S. (2018). Characteristics of outpatient falls that occurred in hospital. Nagoya journal of medical science, $80(3), 417$.

Leiva, A. M., Troncoso-Pantoja, C., Martínez-Sanguinetti, M. A., Petermann-Rocha, F., Poblete-Valderrama, F., Cigarroa-Cuevas, I., \& Celis-Morales, C. (2019). Factores asociados a caídas en adultos mayores chilenos: evidencia de la Encuesta Nacional de Salud 2009-2010. Revista médica de Chile, 147(7), 877886.

Luzia, M. D. F., Cassola, T. P., Suzuki, L. M., Dias, V. L. M., Pinho, L. B. D., \& Lucena, A. D. F. (2018). Incidência de quedas e ações preventivas em um Hospital Universitário. Revista da Escola de Enfermagem da USP, 52.

Luzia, M. D. F., Lucena, A. D. F., \& Argenta, C. (2016). Resultado de enfermagem" Conhecimento: prevenção de quedas": definições conceituais e operacionais de seus indicadores na avaliação de pacientes com diagnóstico de enfermagem risco de quedas em ambiente hospitalar. Clinical and biomedical research. Porto Alegre. 
Research, Society and Development, v. 10, n. 17, e145101724627, 2021

(CC BY 4.0) | ISSN 2525-3409 | DOI: http://dx.doi.org/10.33448/rsd-v10i17.24627

Luzia, M. D. F., Prates, C. G., Bombardelli, C. F., Adorna, J. B., \& Moura, G. M. S. S. D. (2019). Características das quedas com dano em pacientes hospitalizados. Revista gaucha de enfermagem, 40.

Mendes, K. D. S., Silveira, R. C. D. C. P., \& Galvão, C. M. (2019). Uso de gerenciador de referências bibliográficas na seleção dos estudos primários em revisão integrativa. Texto \& Contexto-Enfermagem, 28.

Moreira, P. N. D. O. (2017). Conduta dos enfermeiros na prevenção de queda em idosos em instituições hospitalares (Master's thesis, Brasil).

Neves, A., Melo, A., Mendonça, B., Monteiro, B., Nogueira, D., Barros, E., ... \& Oliveira, V. (2016). FATORES de risco relacionados à queda entre idosos em uma instituição pública de um município do estado de Goiás. Revista Eletrônica Faculdade Montes Belos, 9(1).

Neves, V. L. S., Campos, R. B. N., Da Silva, F. A. A., \& Ribeiro, I. P. (2017). Risco de queda em idosos: instrumento de avaliação. Revista uningá review, 30(2).

Remor, C. P., Cruz, C. B., \& Urbanetto, J. D. S. (2014). Análise dos fatores de risco para queda de adultos nas primeiras 48 horas de hospitalização. Revista Gaúcha de Enfermagem, 35, 28-34.

Santos, M. O., das Graças Aragão, M., Servo, M. L. S., \& de Góis, R. M. O. (2017, December). Indicador de Qualidade Assistencial em Enfermagem na Prevenção de Quedas: um Estudo de Revisão Bibliográfico de 2006 a 2016. In Congresso Internacional de Enfermagem (Vol. 1, No. 1).

Sarges, N. D. A., Santos, M. I. P. D. O., \& Chaves, E. C. (2017). Avaliação da segurança do idoso hospitalizado quanto ao risco de quedas. Revista Brasileira de Enfermagem, 70, 860-867.

Sato, N., Hase, N., Osaka, A., Sairyo, K., \& Katoh, S. (2018). Falls among hospitalized patients in an acute care hospital: analyses of incident reports. The Journal of Medical Investigation, 65(1.2), 81-84.

Silva, D. I. S. D. (2016). A opinião da equipe de enfermagem sobre o uso do protocolo de prevenção de quedas em um hospital público federal. Dissertação [Mestrado em Enfermagem] - Escola de Enfermagem, 2016

Silva-Batalha, E. M. S. D., \& Melleiro, M. M. (2015). Cultura de segurança do paciente em um hospital de ensino: diferenças de percepção existentes nos diferentes cenários dessa instituição1. Texto \& Contexto-Enfermagem, 24, 432-441.

Vaccari, É., Lenardt, M. H., Willig, M. H., Betiolli, S. E., \& de Andrade, L. A. S. (2016). Segurança do paciente idoso e o evento queda no ambiente hospitalar. Cogitare Enfermagem, 21(5).

Vitor, A. F., \& de Araújo, T. L. (2011). Definições para o resultado de enfermagem comportamento de prevenção de quedas: uma revisão integrativa. Revista Eletrônica de Enfermagem, 13(2), 313-22.

Vitorino, L. M., Teixeira, C. A. B., Boas, E. L. V., Pereira, R. L., Santos, N. O. D., \& Rozendo, C. A. (2017). Medo de cair em idosos residentes no domicílio: fatores associados. Revista da Escola de Enfermagem da USP, 51. 